\title{
Silica-poly(styrenesulphonic acid) nanocomposites as promising acid catalysts
}

\author{
M. José Campos Molina, J.J. Corral-Pérez, R. Mariscal and M. López Granados* \\ Institute of Catalysis and Petrochemistry (CSIC), C/ Marie Curie 2, Campus de \\ Cantoblanco, Madrid 28049, (Spain)
}

* Corresponding author: Manuel López Granados

Phonenumber: + 34915854937

Fax number: +34 915854760

e-mail: $\underline{\text { mlgranados@icp.csic.es }}$

\begin{abstract}
Silica-poly(styrene sulphonic acid) nanocomposites ( $\mathrm{SiO}_{2}$-PSSA) were prepared using sol-gel methodologies. An amino functionalized organosilane (APTES) provides the anchoring sites for the retention of this acid polymer by the silica particles network. Aiming at optimizing the polymer loading and stability, different $\mathrm{SiO}_{2}$-PSSA nanocomposites were prepared by changing the type of solvent used in the synthesis, the aging protocol of the gel and the relative concentration of APTES. The effect of these modifications on the catalytic properties of the nanocomposites was explored in two different reactions driven by acid catalysts: xylose dehydration to furfural and esterification of oleic acid with methanol to yield biodiesel. Both reactions are of interest in the valorization of biomass and represent two illustrative cases to exploring the hydrothermal stability of the $\mathrm{SiO}_{2}$-PSSA nanocomposites under different conditions: aqueous phase at moderately high reaction temperatures $(453 \mathrm{~K})$ and organic medium (methanol and oil) at low reaction temperature (333 K), respectively. Polymer leaching was very intense in the case of furfural reaction because of the severe reaction conditions. On the contrary, for the case of the esterification of oleic acid, a reaction conducted under milder conditions, the deactivation by leaching is significantly prevented.
\end{abstract}

Keywords: polymer-SiO${ }_{2}$ composites, xylose, furfural, biodiesel 


\section{Introduction}

Soluble polymers have found applications as catalysts in a very wide number of reactions [1]. Of special interest are those directed at transforming biomass to valuable chemicals and biofuels [2-4] as this strategy will have a definitive impact in the substitution of an economy based in oil by another based on renewable resources. Thus polymers like sulfonated hyperbranched poly(aryleneoxindole)s [5] and poly(styrene sulphonic acid) (hereinafter referred as PSSA) [6] have been tested in reactions for valorization of biomass to valuable products, like cellulose conversion to levulinic acid, biodiesel synthesis, xylose to furfural transformation and oxidation with hydrogen peroxide of furfural to maleic and succinic acid. The former polymers presented acid sites required to catalyse those reactions and moreover were soluble in the reaction medium (water or methanol). Consequently they presented the advantages of homogenous catalysts because the access of reactants to the active sites (and the way out of products) is easier than in solid catalysts. In addition they can be reutilized by separation of the reaction mixture by ultrafiltration membranes.

Other technologies can also be used to recover the polymer as dialysis or precipitation/recrystallization but all are perceived as cumbersome, not simple and cost effective for practical applications. Heterogenisation of the soluble polymers on inorganic solid supports can be an attractive solution. The so formed polymer composite (nanocomposites when the size of the particles of inorganic solid is in the range of $\mathrm{nm}$ ) combines the advantages of soluble polymers (polymer will still be solvated by the liquid) and of the inorganic solids (ease of separation by more ordinary procedures like centrifugation or conventional filtration).

Within this context, the synthesis of $\mathrm{SiO}_{2}$-PSSA nanocomposites has been achieved by polymerization of monomers like styrene or styrene sulphonic acid on the surface of the $\mathrm{SiO}_{2}[7,8]$. However we have followed a different approach and explored the preparation of these polymer-silica nanocomposites by anchoring the pre-formed sulphonated polymer on amino functionalized $\mathrm{SiO}_{2}$ xerogel. Tetraethyl orthosilicate (TEOS) and 3-aminopropyl(triethoxysilane) (APTES) were used as source of $\mathrm{SiO}_{2}$. A sol-gel method was used to obtain a silica xerogel through hydrolysis-condensation of the organosilanes (see scheme 1). PSSA provides itself the acid $\mathrm{pH}$ required to form the $\mathrm{SiO}_{2}$ gel and APTES provides the amino groups. The acid-base reaction between the 
sulphonic acid and the amino groups of the aminofunctionalised silica forms ionic interactions responsible of the retention of the polymer by the silica $[9,10]$. In our case an almost fully sulphonated PSSA was used (y, the fraction of non sulphonated rings, is smaller than 0.05; consequently $x$, the fraction of sulphonated rings, is >0.95). An at. N/S ratio (APTES/PSSA mol ratio) much smaller than 1 was selected for the synthesis. Therefore $m$ (the fraction of sulphonic groups supposedly anchored on amino groups) should be much smaller than $n$ (the fraction non anchored on amino groups; $\mathrm{m}+\mathrm{n}=\mathrm{x}$ ) and therefore free acid sites should be available.

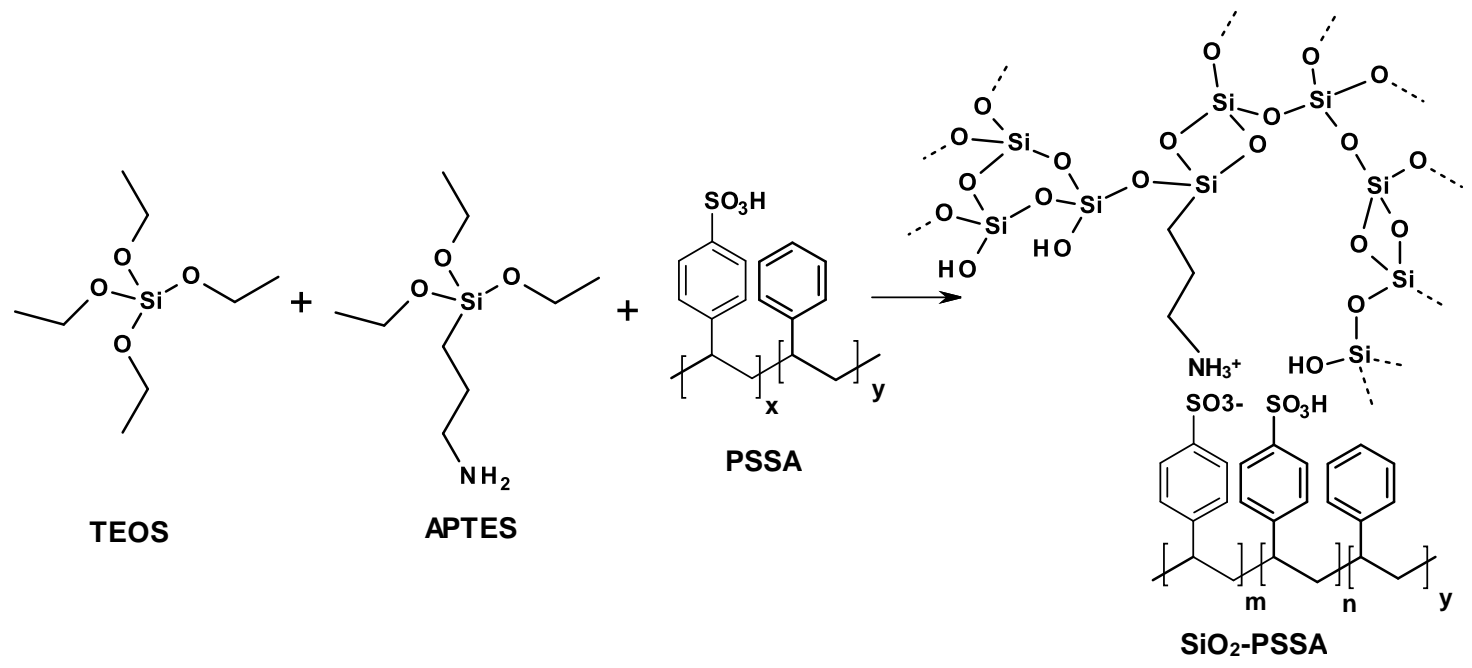

Scheme 1. Schematic representation of the preparation of the $\mathrm{SiO}_{2}-\mathrm{PSSA}$ nanocomposites by the sol-gel methodology used in this study

As indicated above, the approach described in Scheme 1 makes use of already prepared PSSA. Interestingly PSSA can be obtained from polystyrene (PS) by sulphonation and, actually, we have also demonstrated that in practice PS waste can be sulphonated to form PSSA and be used as catalyst (Waste-To-Catalyst concept, WTC) [11]. Consequently there is no need of using fresh monomers to prepare this type of acid catalysts but to recycle PS waste.

In an attempt of finding the best preparation conditions in terms of polymer loading (acid sites loading) and hydrothermal stability, we have previously explored the effect of two variables of synthesis: temperature of synthesis and the $\mathrm{S} / \mathrm{N}$ at. ratio initially used for the preparation (PSSA/APTES ratio). The rest of variables of synthesis were kept constant. We have found that an at. $\mathrm{S} / \mathrm{N}$ ratio between 4.4-8.8 and a temperature of synthesis $=348 \mathrm{~K}$ resulted in nanocomposites with the largest acid sites 
loading and with the best hydrothermal properties in terms of stability of the catalyst [12]. However when the nanocomposites were used in the xylose to furfural reaction, the catalysts deactivated upon reutilization, mainly because of an intense polymer leaching.

Consequently improvement of the hydrothermal stability is still needed. And moreover it is also interesting to explore other possible catalytic applications of these nanocomposites. In the investigation presented here, we report the effect of other variables of synthesis on the chemical and catalytic behavior of $\mathrm{SiO}_{2}$-PSSA nanocomposite. These variables were the utilization of methanol as solvent to prepare the nanocomposite (instead of water), the time of aging of the gel, the slow evaporation of the water before the drying of the gel and the N/Si at. ratio used during the synthesis.

Besides the xylose to furfural reaction, we have extended the study of the catalytic activity to the esterification of fatty acids with methanol to render fatty acid methyl esters (FAME). Both reactions are of interest in the valorization of biomass to renewable chemicals and to biofuels $[6,10,11,13]$. Furfural has been considered a valuable bio-derived chemical platform for a number of other commodities and biofuels [14]. The esterification of fatty acid to FAMEs is a key reaction for the valorization of low quality oils to biodiesel [15-17]. In addition to these practical reasons, these two reactions represent two extreme cases when exploring the hydrothermal stability of the $\mathrm{SiO}_{2}$-PSSA nanocomposites: aqueous phase at moderately high reaction temperatures, on one side, and organic medium (methanol and oil) at low reaction temperature, on the other side. Therefore with these two reactions we are delimiting the type of reaction this type catalyst can be applied for. We have paid specific attention to the reusability of the nanocomposites and, specifically, to the stability against leaching of the polymer.

\section{Experimental}

\subsection{Preparation of catalysts}

The nanocomposites were prepared by sol-gel methodology by co-condensation of tetraethyl orthosilicate (TEOS, Sigma-Aldrich, $\geq 99 \%$ ) and (3-aminopropyl) triethoxysilane (APTES, Sigma-Aldrich, $\geq 98 \%$ ) in the presence of PSSA (MW = $75000,5.4 \mathrm{mmoles} \cdot \mathrm{H}^{+} \cdot \mathrm{g}^{-1}$ ) according to a methodology described elsewhere [10]. PSSA was supplied by Sigma-Aldrich as a $18 \mathrm{wt}$ \% aqueous solution. The required amounts of 
the aqueous PSSA solution, TEOS and APTES were introduced in a round glass flask vessel and the mixture stirred vigorously. The reactor was immersed into an oil bath at $348 \mathrm{~K}$ and kept at this temperature for $16 \mathrm{~h}$ (overnight). The solid so formed after aging was filtrated and to fully remove the weakly retained polymer, the solid was subjected to successive washes with water at room temperature until rinsing water displayed neutral pH. Finally, the solid was dried in air at $393 \mathrm{~K}$ for $16 \mathrm{~h}$.

In a first series of samples the initial amount of APTES and TEOS were varied as to have an atomic N/Si ratio of 0.05, 0.09 and 0.20 respectively, maintaining constant the PSSA/APTES mol ratio ( $\mathrm{S} / \mathrm{N}=4.4)$. In practice ca. $5 \mathrm{~g}$ of APTES, ca. $18.3 \mathrm{~g}$ of PSSA solution and ca. 89.4, 47.1 and $18.8 \mathrm{~g}$ of TEOS, respectively, were incorporated to the synthesis reactor. The nanocomposites of this series were named as $\mathrm{Si}$-(N/Si)PSSA-4.4 where N/Si is refers to the ratio used in the synthesis whereas 4.4 is the initial at. $\mathrm{S} / \mathrm{N}$ ratio. We kept the ratio $\mathrm{S} / \mathrm{N}=4.4$ in the labeling to distinguish them from the other nanocomposites investigated in this article.

The effect of the utilization of methanol instead of water as the medium for the synthesis was also explored. APTES and TEOS are not soluble in water and therefore when using water two phases exist initially: the organic layer with APTES and TEOS and the aqueous solution of PSSA. On the contrary APTES, TEOS and PSSA are soluble in methanol and a single phase is available from the very beginning. $5 \mathrm{~g}$ of dried PSSA solid, ca. $9.18 \mathrm{~g}$ of water (the amount required to fully hydrolyze TEOS and APTES), ca. $25.7 \mathrm{~g}$ of TEOS and ca. $2.7 \mathrm{~g}$ of APTES were incorporated to the synthesis flask that was earlier filled with ca. $100 \mathrm{ml}$ of methanol, enough to solubilize all reactants. The gel formed was aged for $16 \mathrm{~h}$ at $348 \mathrm{~K}$. Then the solid was filtered, washed and dried as indicated above.

A modification of the previous Si-0.09-PSSA-4.4 sample was prepared by extending the aging period with respect to that previously used: instead of aging at 348 $\mathrm{K}$ for $16 \mathrm{~h}$, gel was aged in contact with the mother liquor for 1 week. In practice, ca.10 $\mathrm{g}$ of PSSA, TEOS $(25.7 \mathrm{~g})$ and APTES $(2.7 \mathrm{~g})$ were introduced in the reactor and left aging for one week at $348 \mathrm{~K}$. Then the solid was filtrated, washed and dried as indicated above.

A last sample was prepared by subjecting the gel aged for $16 \mathrm{~h}$ at $348 \mathrm{~K}$ to a second aging period at $333 \mathrm{~K}$ and allowing both the water and ethanol form by the hydrolysis of the organosilanes to slowly evaporate until dryness for one day. The 
resulting solid was dried as usual at $393 \mathrm{~K}$ for $16 \mathrm{~h}$. In this case a sample corresponding to an atomic S/N ratio and N/Si ratio of 24 and 0.09 , respectively was synthesized. An initial amount of polymer much larger than previously used was used aiming at preparing a nanocomposite with a larger loading of polymer. This catalyst is referred as Si-0.09-PSSA-24-ev where 0.09 and 24 refers, respectively, to the N/Si and S/N initially used during the synthesis and $e v$ ending refers to the fact that solvents in the mother liquor has been removed before drying. For comparison purposes a blank sample was prepared without the slow evaporation, following the same N/Si and S/N ratios: once the gel was formed and aged for $16 \mathrm{~h}$ at $348 \mathrm{~K}$ it was filtered, washed and dried at 373 $\mathrm{K}$ as usual. It was named Si-0.09-PSSA-24.

Other chemicals used in the experiments were $D(+)$-xylose $(\geq 99 \%)$, 2furaldehyde reagent grade (99\%), cyclopentyl methyl ether (CPME) ( $\geq 99 \%)$, octanoic acid (98\%), oleic acid (90\%) and acetonitrile ( $299.9 \%)$ all supplied by Sigma-Aldrich. Methanol (99.5\%) from Panreac, tetrahydrofuran (THF, HPLC grade) from Scharlau, ethyl palmitate ( $\geq 95 \%$ ) from Fluka and commercial food grade sunflower oil were also used. All materials were used without further purification and mili-Q $\mathrm{H}_{2} \mathrm{O}$ was used for preparation of all aqueous solutions.

\subsection{Characterisation of nanocomposites}

The elemental analysis of the solids $(\mathrm{C}, \mathrm{H}, \mathrm{N}$ and $\mathrm{O}$ concentration) was performed on a LECO CHNS-32 analyzer. Typically, $1 \mathrm{mg}$ of solid was placed in an Ag crucible and combusted at $1333 \mathrm{~K}$ under a pure $\mathrm{O}_{2}$ atmosphere. The $\mathrm{CO}_{2}, \mathrm{H}_{2} \mathrm{O}$ and $\mathrm{SO}_{2}$ gases were quantified by Fourier transform infrared (FT-IR) spectroscopy while $\mathrm{N}_{2}$ was determined by differential thermal conductivity.

The actual Si content of the samples was determined by total reflection X-ray fluorescence (TXRF) using an Atomika 8030C TXRF spectrometer (Cameca Germany) equipped with a $3 \mathrm{~kW} \mathrm{Mo/W}$ dual target X-ray tube and a W/C double monochromator multilayer. A Si (Li) detector with an active area of $80 \mathrm{~mm}^{2}$ and a resolution of $150 \mathrm{eV}$ at $5.9 \mathrm{keV}(\mathrm{Mn} \mathrm{K \alpha})$ was used for detection and measurement of the produced X-rays.

The amount of acid sites of the different polymer hybrids were determined by acid-base titration. In practice a known amount of catalyst $(100 \mathrm{mg})$, previously dried, was put in contact with ca. $25 \mathrm{~mL}$ of an aqueous solution containing $1 \mathrm{~g}$ of $\mathrm{NaCl}$ and stirred at $323 \mathrm{~K}$ for $16 \mathrm{~h}$. The aim was to produce the exchange between protons from sulphonic groups and sodium ions. After that, the solution was filtered and the retained 
solid was repeatedly washed with water. The resulting filtrate solution was titrated with a $0.005 \mathrm{M} \mathrm{KOH}$ solution (previously standardized with dry potassium acid phfhalate). A few drops of an ethanolic solution of phenolphthalein were used to determine the end point.

The ${ }^{13} \mathrm{C}$ and ${ }^{29} \mathrm{Si}$ solid state NMR spectra of the samples were recorded in an AV-400-WB Bruker spectrometer equipped with a tripe channel probe. Powder samples were finely grounded and dried for several days at $373 \mathrm{~K}$ in an oven and then rapidly transferred to $\mathrm{ZrO}_{2}$ rotors $(4 \mathrm{~mm})$ and capped with Kel-F caps to prevent the hydration of the polymer. Frequencies used were 100.32 and $79.49 \mathrm{MHz}$ for ${ }^{13} \mathrm{C}$ and ${ }^{29} \mathrm{Si}$ nuclei, respectively. Samples were spun at $10 \mathrm{kHz}$. The CP-MAS ${ }^{1} \mathrm{H}-{ }^{13} \mathrm{C}$ spectra were recorded by using a spectral width of $35 \mathrm{KHz}$, excitation pulse for ${ }^{1} \mathrm{H}$ of $3,4 \mu \mathrm{s}$, contact time of $3,5 \mathrm{~ms}$ and a relaxation time of $4 \mathrm{~s}$, with ${ }^{1} \mathrm{H}$ tppm 15 decoupling at $80 \mathrm{KHz}$. The number of scans was 1024 for ${ }^{13} \mathrm{C}$ spectra. ${ }^{13} \mathrm{C}$ chemical shift is referenced to the adamantane $\mathrm{CH}_{2}$ signal (29.5 ppm) as secondary reference relative to the TMS as a primary reference. ${ }^{29} \mathrm{Si}$ MAS-NMR spectra are the result of 1000 accumulations after direct irradiation at a spectral width of $15 \mathrm{KHz}$, a relaxation delay of $60 \mathrm{~s}$ and pulses of $\pi / 4$ at $50 \mathrm{KHz}$. Kaolin signal (-91.2 ppm) was used like secondary reference relative to a TMS as primary reference.

\subsection{Catalytic activity of nanocomposites}

The catalytic experiments of xylose dehydration were performed in a $15 \mathrm{~mL}$ Ace sealed pressure glass reactor flushed with nitrogen before use, magnetically stirred (1000 rpm) and placed in a preheated oil bath at a selected temperature. In practice, 1.35 $\mathrm{g}$ of water, $3.5 \mathrm{~g}$ of CPME as organic solvent (2.33 g CPME/g aqueous solution), 150 $\mathrm{mg}$ of xylose (10 wt. \% with respect to water) and $75 \mathrm{mg}$ of finely grounded catalyst previously sieved to a size $<106 \mu \mathrm{m}$ (5 wt. \% with respect to water, catalyst/xylose wt. ratio $=0.5$ ) were poured into the reactor. CPME solvent has been reported as an excellent solvent to improve furfural yield [13]. The reaction is stopped by removing the reactor from the oil bath and rapidly cooled down by immersion in water at room temperature. Then, internal standard for organic phase (40 mg of octanoic acid) was added to the quenched reaction mixture and after gently agitation for several minutes, mixture was centrifuged to separate both phases. A $2 \mathrm{~mL}$ aliquot was taken from the organic layer. Once the organic phase has been aliquoted, internal standard for aqueous 
phase (200 mg of D (+)-glucose) was added to the left reaction mixture and, after agitation for several minutes and centrifugation, an aliquot (ca. $2 \mathrm{~mL}$ ) was taken from the aqueous layer for analysis. Both organic and aqueous aliquots were filtered $(0.22$ $\mu \mathrm{m})$ to remove the catalyst and the solid humins formed during the reaction. Aqueous aliquots were analyzed with a HPLC Agilent 1200 series chromatograph equipped with a refraction index (RI) detector and a Bio-Rad Aminex HPX-87H column (300x7.8 $\mathrm{mm}$ ) for analysis of xylose and furfural in aqueous phase. A $0.005 \mathrm{M} \mathrm{H}_{2} \mathrm{SO}_{4}$ mobile phase was employed as eluent with $0.4 \mathrm{~mL} / \mathrm{min}$ flow rate and at $328 \mathrm{~K}$. In the case of organic aliquots, analysis of furfural in this phase was conducted by gas chromatography (CG) (Varian CP-3800) equipped with a ZBWAX plus column (30mx0.32mmx0.25um) and a flame ionization detector (FID).

The catalytic experiments of esterification of oleic acid were performed in the same Ace sealed pressure glass reactor magnetically stirred $(1000 \mathrm{rpm})$ and placed in a preheated oil bath at a selected temperature $(333 \mathrm{~K})$. For a typical experiment $0.872 \mathrm{~g}$ of methanol, $2 \mathrm{~g}$ of sunflower oil (methanol/oil mol ratio = 12), $0.1 \mathrm{~g}$ of oleic acid (FFA/oil wt. ratio $=0.05$ ) and $40 \mathrm{mg}$ of finely grounded catalyst $(3.3 \mathrm{wt} \%$ respect to the total solution, catalyst/FFA wt. ratio $=0.4$ ) were poured into the reactor. Then, internal standard $(0.1 \mathrm{~g}$ of ethyl palmitate in THF) was added to the quenched reaction mixture and after gently agitation for several minutes, mixture was centrifuged. A $2 \mathrm{~mL}$ aliquot was taken from the solution and filtered $(0.22 \mu \mathrm{m})$ to remove the solid. Then, this aliquot was analyzed with a HPLC Agilent 1200 series chromatograph equipped with a refraction index (RI) detector and a XDB-C18 column $(5 \mu \mathrm{m}, 4.6 \times 150 \mathrm{~mm})$ for analysis of all the reactants and products. A solution of $5 \% \mathrm{v} / \mathrm{v}$ of THF in acetonitrile mobile phase was employed as eluent with $0.6 \mathrm{~mL} / \mathrm{min}$ flow rate and at $308 \mathrm{~K}$.

\section{Results and Discussion}

\subsection{Characterisation of the nanocomposites}

\subsubsection{Chemical and acidity analyses}

The Si-(N/Si)-PSSA-4.4 series

Table 1 shows the experimental concentration of the relevant atoms of these nanocomposites (first three rows). For the $\mathrm{S}$ and $\mathrm{N}$ loading, the theoretical values that 
should be obtained considering the amounts of TEOS, APTEOS and PSSA used in the synthesis have been incorporated between brackets to the corresponding columns. The experimental acid sites loading has been also included (fourth columns), in this column the difference between the $\mathrm{S}$ and $\mathrm{N}$ loading is also compiled between brackets. Finally the fifth column informs on the experimental atomic $\mathrm{S} / \mathrm{N}$ ratio and the theoretical values deduced from the amounts used during the synthesis also appear between brackets. The concentration of $\mathrm{S}$ in the nanocomposites $\left(\mathrm{mmol} \mathrm{S} \cdot \mathrm{g}_{\mathrm{cat}}{ }^{-1}\right)$, and consequently the amount of polymer retained by the xerogel, increases as $\mathrm{N} / \mathrm{Si}$ increases. This is reasonable because the relative amount of APTES increases with respect to that of TEOS and therefore there will be more amino groups available for anchoring the polymer molecules. However the amount of retained polymer is in practice smaller than that theoretically expected (see the value between brackets in the mmol S. $\mathrm{g}_{\text {cat }}{ }^{-1}$ column) indicating that part of the polymer is not retained and is leached away during synthesis. Consequently, since there is fewer polymer than expected, the $\mathrm{N}$ concentration is larger than that theoretically expected (see column mmolN. $\mathrm{g}_{\text {cat }}{ }^{-1}$ where these tow data are compiled). On the other hand, it is worth to notice that the experimental N/Si ratio is quite close to the nominal one. Therefore both atoms have been incorporated to the solid with the same ratio they were in the initial preparation mixture. This strongly suggests that there was no selective hydrolysis of one of the precursor with respect to the other and that all $\mathrm{N}$ has been incorporated to the solid.

Table 1. Experimental concentration of relevant atoms in the relevant nanocomposites

\begin{tabular}{|c|c|c|c|c|c|}
\hline Name & mmol S·g ${ }_{\text {cat }}^{-1}$ & $\mathrm{mmolN} \cdot \mathrm{g}_{\text {cat }}{ }^{-1}$ & $\mathrm{mmol} \mathrm{H}^{+} \cdot \mathrm{g}_{\mathrm{cat}}{ }^{-1 \mathrm{~b}}$ & at. $\mathbf{N} / \mathrm{Si}^{\mathrm{c}}$ & at. $\mathbf{S} / \mathbf{N}$ \\
\hline Si-0.05-PSSA-4.4 & $1.22(2.14)^{\mathrm{a}}$ & $0.52(0.49)^{\mathrm{a}}$ & $0.59(0.70)^{\mathrm{d}}$ & $0.05(0.05)^{\mathrm{a}}$ & $2.3(4.4)^{\mathrm{a}}$ \\
\hline Si-0.09-PSSA-4.4 & $1.92(2.90)^{\mathrm{a}}$ & $0.73(0.66)^{\mathrm{a}}$ & $0.99(1.19)^{\mathrm{d}}$ & $0.09(0.09)^{\mathrm{a}}$ & $2.6(4.4)^{\mathrm{a}}$ \\
\hline Si-0.20-PSSA-4.4 & $2.43(3.82)^{\mathrm{a}}$ & $1.29(0.87)^{\mathrm{a}}$ & $0.88(1.14)^{\mathrm{d}}$ & $-(0.2)^{\mathrm{a}}$ & $1.9(4.4)^{\mathrm{a}}$ \\
\hline Si-0.09-PSSA-24 & $1.88(4.68)^{\mathrm{a}}$ & $0.83(0.19)^{\mathrm{a}}$ & $0.94(1.05)^{\mathrm{d}}$ & $0.08(0.09)^{\mathrm{a}}$ & $2.27(24)^{\mathrm{a}}$ \\
\hline $\begin{array}{c}\text { Si-0.09-PSSA-24- } \\
\text { ev }\end{array}$ & $4.49(4.68)^{\mathrm{a}}$ & $0.25(0.19)^{\mathrm{a}}$ & $4.03(4.24)^{d}$ & $0.12(0.09)^{\mathrm{a}}$ & $\begin{array}{l}17.92 \\
(24)^{\mathrm{a}}\end{array}$ \\
\hline \multicolumn{6}{|c|}{${ }^{\mathrm{a}}$ Values between brackets refers to nominal concentration incorporated in the preparation mixture } \\
\hline
\end{tabular}


The experimental $\mathrm{S}$ concentration is larger than that of $\mathrm{N}$ and therefore there are more sulphonic groups than amino groups. As a consequence non neutralized sulphonic sites are available and solid present acid sites. In principle since the amount of polymer increases upon N/Si nominal ratio, there would be more acid sites. However the actual acid sites concentration reaches a maximum for Si-0.09-PSSA-4.4 $\left(0.99 \mathrm{mmol} \mathrm{H}^{+} \cdot \mathrm{g}_{\text {cat }}{ }^{-1}\right)$ and slightly become smaller for Si-0.2-PSSA-4.4 $\left(0.88 \mathrm{mmol} \mathrm{H}^{+} \cdot \mathrm{g}_{\mathrm{cat}}{ }^{-1}\right)$. The reason is that the $\mathrm{N}$ concentration also increases within this series and for the latter sample the relative increase of $\mathrm{N}$ concentration $\left(1.29 \mathrm{mmol} \mathrm{N} \cdot \mathrm{g}_{\mathrm{cat}}{ }^{-1}\right)$ is larger than that of the $\mathrm{S}$ loading. In consequence there are less free sulphonic sites (not neutralized by amino groups).

\section{Type of solvent and time aging the gel}

Changing water for methanol in the synthesis did not result in any increase of the amount of polymer retained by the silica xerogel. Same conclusion was reached when the gel was aged for one week (instead that for $16 \mathrm{~h}$ ) in the mother aqueous solution. None of these modifications are useful for improving the loading of acid sites. These samples were discarded for further studies.

\section{Effect of evaporation of the water before drying}

In this case, instead of filtering the solid formed after aging for $16 \mathrm{~h}$ at $348 \mathrm{~K}$, water and ethanol (the latter formed from the hydrolysis of organosilanes) were gently removed at $333 \mathrm{~K}$ by evaporation at mild temperature. The solid formed after evaporation was subsequently dried in air as usual ( $393 \mathrm{~K}$ for $16 \mathrm{~h}$ ). The intention of the evaporation is that while drying, gel shrinks and collapses and polymer can be trapped by entangling in the network of the gel particles what may be of help to firmly retaining the polymer (besides the electrostatic interactions). Additionally, in an attempt of increasing the polymer loading in the resulting nanocomposite, very high polymer concentration is used for the synthesis $(\mathrm{S} / \mathrm{N}=24)$. A blank nanocomposite was also prepared but without gently evaporation of the solvents before drying at $393 \mathrm{~K}$. The experimental concentrations of the relevant atoms for these samples are also tabulated in Table 1 ( $4^{\text {th }}$ and $5^{\text {th }}$ rows). 
Blank Si-0.09-PSSA-24 nanocomposite presents $\mathrm{S}$ and acid sites concentration similar to Si-0.09-PSSA-4.4 one. The incorporation of more PSSA to the preparation mixture did not improve the polymer loading, indicating that a large fraction of the polymer is not retained by the functionalized silica and it is leached during the filtration step. On the contrary the Si-0.09-PSSA-24 nanocomposite, prepared by slow evaporation, presents a very large $\mathrm{S}$ and acid sites concentration; we have to bear that this nanocomposite was not subjected to a filtration step during preparation and therefore all polymer used for the preparation must be in the solid. The question that still remains is whether the slow evaporation can result in the stabilization of the polymer (entangled within the network of silica aging particles) and consequently in a nanocomposite with better hydrothermal stability against leaching. This question will be later answered when studying the stability and reusability of the nanocomposite in the reactions investigated in this article.

\subsubsection{NMR studies}

Figure 1 displays the ${ }^{29} \mathrm{Si}$ MAS-NMR and ${ }^{13} \mathrm{C}$ CP-MAS-NMR spectra of the most representative nanocomposites prepared. Regarding the ${ }^{29} \mathrm{Si}$ spectra the broad resonance at $-101 \mathrm{ppm}$ is assigned to $\mathrm{Q}^{3}$ siloxane species of the silica framework and appears in all $\mathrm{Si}-(\mathrm{N} / \mathrm{Si})-\mathrm{PSSA}-4.4$ series samples $\left(\left[\mathrm{Q}^{\mathrm{n}}=\mathrm{Si}(\mathrm{OSi})_{\mathrm{n}}(\mathrm{OR})_{4-\mathrm{n}}\right]\right.$ where $\mathrm{n}=3$ and $\mathrm{R}=$ alkoxy or $\mathrm{H})$ [18-21]. $\mathrm{Q}^{3}$ resonance mostly originates from $(\mathrm{SiO})_{3} \mathrm{Si}-\mathrm{OH}$ units (according to previous DRIFT studies the amount of unhydrolized ethoxy residues must be quite low [12]). The signal at $-110 \mathrm{ppm}$ corresponds $\mathrm{Q}^{4}$ species $\left(\mathrm{n}=4, \mathrm{Q}^{4}\right.$ represents $\mathrm{Si}$ atoms in fully condensed $\mathrm{Si}(\mathrm{O}-\mathrm{Si})_{4}$ tetrahedra). The latter signal becomes relatively more intense for the sample with the larger N/Si (0.09 and 0.2) indicating that in these
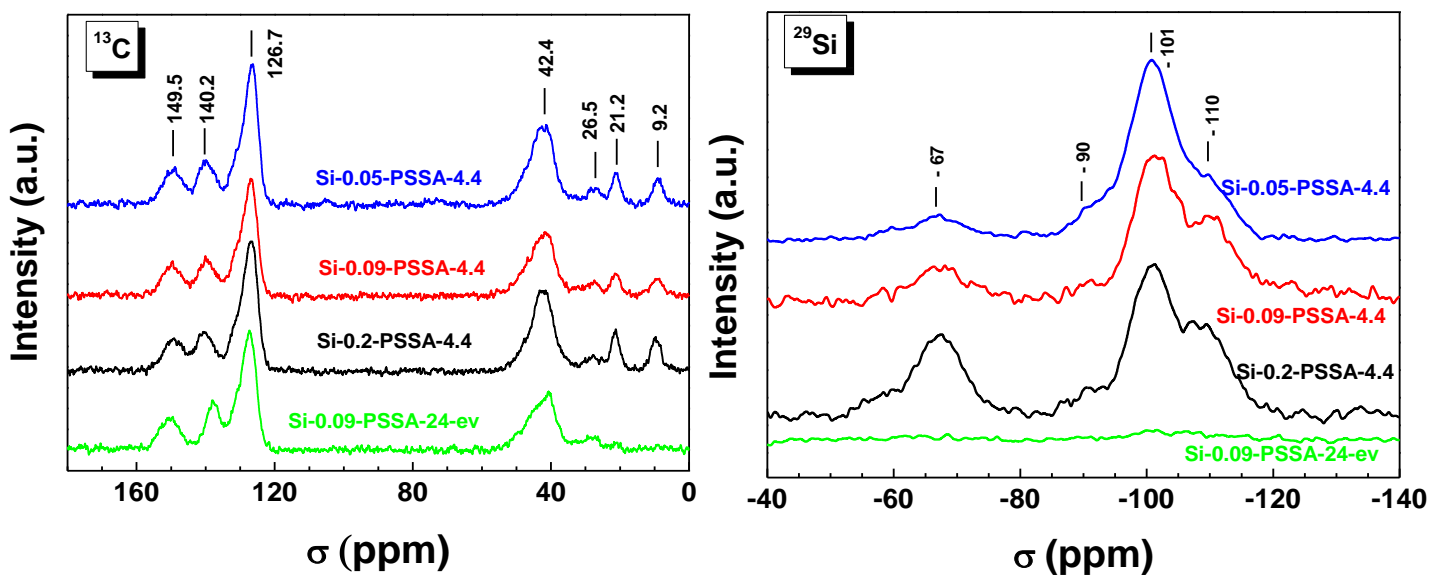

Figure $1 .{ }^{29} \mathrm{Si}$ MAS-NMR and ${ }^{13} \mathrm{C} \quad \mathrm{CP}-\mathrm{MAS}-\mathrm{NMR}$ spectra of nanocomposites 
samples the silica framework is more condensed. On the other hand, a weak $Q^{2}(n=2)$ signal at $-90 \mathrm{ppm}$ (originating from $(\mathrm{SiO})_{2} \mathrm{Si}-(\mathrm{OH})_{2}$ like species) is also incipiently visible. These all latter signals were of very low intensity for the Si-0.09-PSSA-24-ev sample, in agreement with chemical analyses that showed that this sample is very concentrated in polymer.

The detection in all the fresh samples of the resonance at -67 ppm (weak although observable) indicates the presence of aminopropyl functionalized Si atoms [19, 22]. This signal corresponds to alkylsiloxane $T^{3}$ units $\left(\mathrm{R}^{\prime} \mathrm{Si}(\mathrm{OSi})_{3}\right)$, where $\mathrm{R}^{\prime}$ corresponds to aminopropyl group. The presence of $\mathrm{T}^{2}$ species $\mathrm{R}^{\prime}(\mathrm{SiO})_{2}(\mathrm{OH})$ cannot be discarded as the signal is $\mathrm{T}^{3}$ signal is quite wide and presents an incipient shoulder at lower shifts assignable to $\mathrm{T}^{2}$ signals. The intensity of this signal is smaller for Si-0.09PSSA-4.4 and Si0-0.05-PSSA-4.4 catalysts, respectively, that is in harmony with the lower $\mathrm{N}$ loading observed for these samples by elemental chemical analysis. The presence of these $\mathrm{T}^{\mathrm{i}}$ signals also demonstrates that aminopropyl groups are present in the samples, in agreement of the detection of $\mathrm{N}$ by chemical analysis

Regarding the ${ }^{13} \mathrm{C}$ spectra of the $\mathrm{Si}-(\mathrm{N} / \mathrm{Si})-\mathrm{PSSA}-4.4$ series, these nanocomposites displayed a similar NMR profile. Four signals coming from the polymer are clearly identified: one at $42.4 \mathrm{ppm}$ from not aromatic $\mathrm{CH}$ and $\mathrm{CH}_{2}$ carbons, other at $140.2 \mathrm{ppm}$ from the aromatic $\mathrm{C}$ atom bearing the sulphonic group, other at $126.7 \mathrm{ppm}$ from aromatic $\mathrm{C}$ linked to the aliphatic chain and finally another signal at $149.5 \mathrm{ppm}$ from the rest of the $\mathrm{C}$ aromatic atom in the aromatic ring [6]. Two additional peaks can be also observed arising from $\mathrm{C}$ atoms of the aminopropyl groups at 9.2 and $21.2 \mathrm{ppm}$ (the carbon atoms bearing the amino group $\left(\mathrm{C}_{\alpha}\right)$ and that at $\beta$ position with respect to the latter $\left(C_{\beta}\right)$, respectively). The signal from $C \gamma$ should be at around 45-50 ppm but is overshadowed by the peak at $42.4 \mathrm{ppm}$ from the polymer. The weak feature at ca. $27 \mathrm{ppm}$ is assigned to a side band of the main signal at $126 \mathrm{ppm}$. In the case of Si0.09-PSSA-24-ev the signals arising from the polymer become predominant whereas those from aminopropyl groups are much weaker. As indicated above for Si signals, this spectrum corresponds to a nanocomposite with a high concentration of polymer and then relatively much less silica, and therefore the intensity of the $\mathrm{C}$ peaks from the aminopropyl groups also are lower.

The $\mathrm{C}_{\alpha}$ and $\mathrm{C}_{\beta}$ resonances of the aminopropyl group are sensitive to protonation. The positions of these peaks correspond to protonated amine groups $\left(\mathrm{R}^{-\mathrm{NH}_{3}}{ }^{+}\right)[10,23$ 25] because those of unprotonated amines are shifted by $c a .2$ and $5 \mathrm{ppm}$, respectively, 
to lower shieldings in unprotonated amines. The protonation results from the interaction with sulphonic groups (protonation from acidic silanols was excluded) [10]. These protonation of the amino groups by acid-base reaction with the sulphonic groups is responsible for the anchoring of the polymer in the solid and for the retention of the polymer.

\subsection{Catalytic activity}

\subsubsection{Catalytic activity of nanocomposites in xylose dehydration to furfural}

Figure 2 displays the catalytic properties of the most relevant nanocomposites studied in this article. The catalysts prepared by changing the solvent or by lengthening the aging period were not included for the sake of simplicity as these modifications did not represent an improvement in the acid loadings. Concerning the $\mathrm{Si}-(\mathrm{N} / \mathrm{Si})-\mathrm{PSSA}-4.4$ series, a rapid perusal of the results shows that the conversion and yield values rises

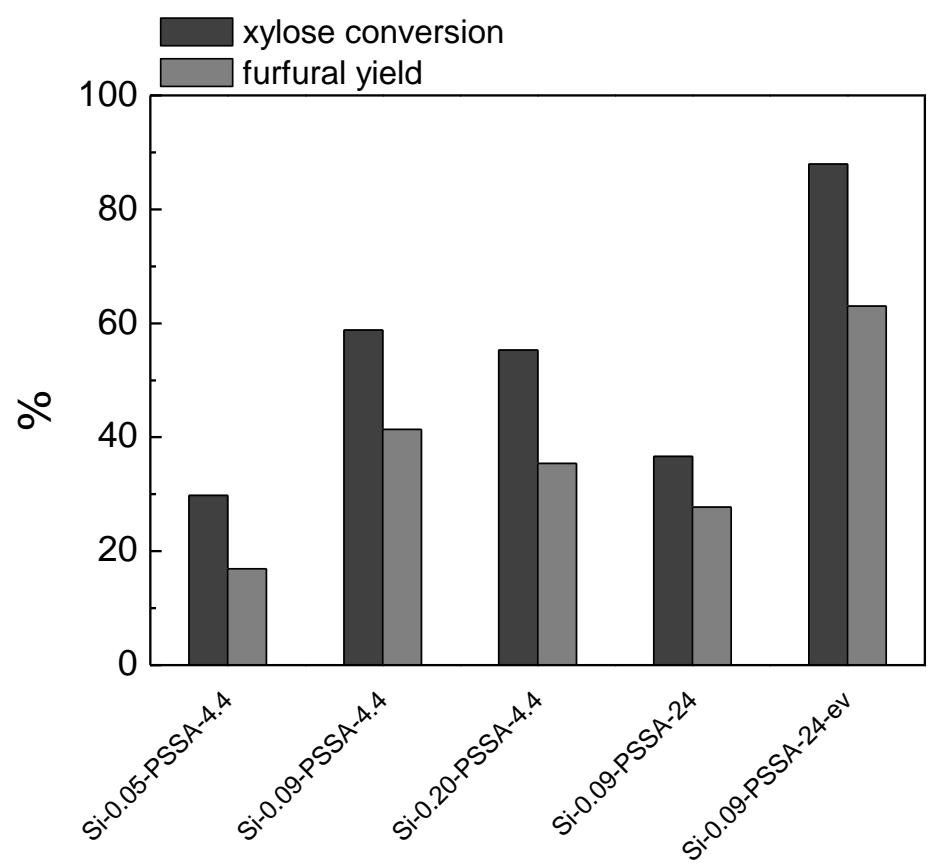

Figure 2. Comparison of xylose conversion and furfural yield for the different catalysts studied. Reaction conditions: $10 \mathrm{wt} . \%$ xylose and $5 \mathrm{wt} . \%$ catalyst in the aqueous phase, CPME/aqueous phase mass ratio $=2.33 ; 5 \mathrm{~g}$ total solution, temperature of reaction $453 \mathrm{~K}$, time of reaction $30 \mathrm{~min}$.

upon the acid sites concentration and that the Si-0.09-PSSA-4.4 sample, with the largest acid sites loading, present the best activity and yield to furfural. So as a first approximation, the number of acid sites (polymer loading) is a key variable defining the 
catalytic properties. The comparison between Si-0.09-PSSA-24 and Si-0.09-PSSA-24ev is also in agreement with this conclusion as the latter presents the highest acid sites concentration among the nanocomposites prepared in this study and so is the catalytic performance.

However the loading of acid sites is not the only property defining the catalytic performance of the nanocomposites: Si-0.09-PSSA-4.4 and Si-0.09-PSSA-24 present similar acid sites loading and their catalytic activity are dissimilar. Texture or accessibility of the reactant molecules to the active sites must be another relevant property. It must be taken into account that polymer is anchored and entangled within a network of silica particles, so porosity and tortuosity must be also considered to define the catalytic activity. Unfortunately texture of nanocomposites cannot be studied as they presented a lack of adsorption of $\mathrm{N}_{2}$ because part of the polymer is also filling the pores and this substantially reduces the $\mathrm{N}_{2}$ adsorption [10].

Figure 3 presents the reutilization of the two most active nanocomposites represented in Figure 2. For the sake of simplicity only furfural yields are represented. A remarkable deactivation was observed for Si-0.09-PSSA-4.4 nanocomposite during the first run, a residual activity is observed for the rest of runs. On the other hand a close
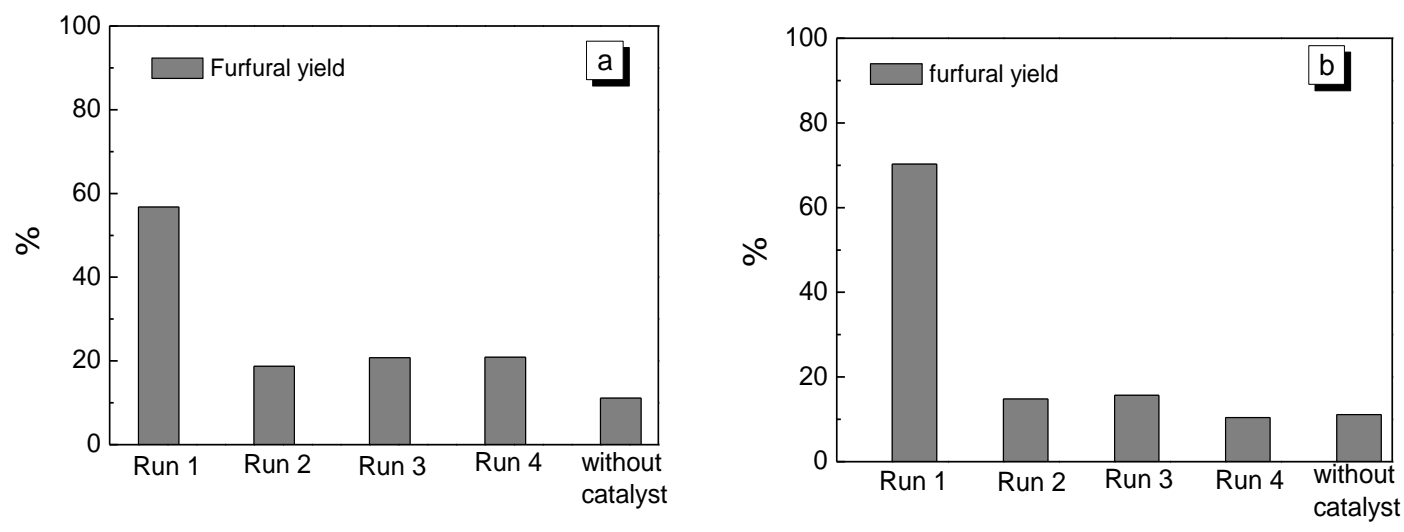

Figure 3. Reutilization of a) Si-0.09-PSSA-4.4 and b) Si-0.09-PSSA-24-ev catalysts Reaction conditions: $10 \mathrm{wt} . \%$ xylose and $5 \mathrm{wt} . \%$ catalyst in the aqueous phase, CPME/aqueous phase mass ratio= 2.33; $5 \mathrm{~g}$ total solution, $453 \mathrm{~K}, 60 \mathrm{~min}$.

to complete deactivation was detected for Si-0.9-PSSA-24-ev. 
A likely cause of deactivation can be the leaching of the polymer during reaction. With the intention of assessing on the leaching, the chemical analyses of the nanocomposites resulting from hydrothermally treating the fresh nanocomposites at 453 $\mathrm{K}$ for $16 \mathrm{~h}$ with the same reaction mixture but without xylose (only CPME and water) were carried out. In practice, $400 \mathrm{mg}$ of each catalyst, 1.5 grams of water and 3.5 grams of CPME $(\mathrm{CPME} /$ aqueous phase mass ratio= 2.33$)$ were poured into the glass reactor where catalytic reactions were conducted and stirred at $453 \mathrm{~K}$ for $16 \mathrm{~h}$. This treatment represents more than five reutilization cycles of figure 3 , so it is a very reasonable approximation to the changes in properties that can occur on the nanocomposites during reutilization. After that, the reaction mixture was cooled and centrifuged to separate the liquid from the solid catalyst. The solid was then thoroughly washed again with water to remove all not retained polymer. Once the liquid was centrifuged, the treated solid was dried at $393 \mathrm{~K}$ for $16 \mathrm{~h}$ and the $\mathrm{S}$ and acid site loading determined as explained above. Thus Si-0.09-PSSA-4.4 presented $1.28 \mathrm{mmol} \mathrm{S} \cdot \mathrm{g}_{\text {cat }}{ }^{-1}$ and $0.22 \mathrm{mmolH}^{+} \cdot \mathrm{g}_{\text {cat }}{ }^{-1}$ after this hydrothermally treatment, representing respectively $67 \%$ and $22 \%$ of the values of fresh sample (see table 1). Si-0.9-PSSA-24-ev nanocomposite after the hydrothermal treatment presented $1.77 \mathrm{mmol} \mathrm{S} \cdot \mathrm{g}_{\mathrm{cat}}{ }^{-1}$ and $0.69 \mathrm{mmolH}^{+} \cdot \mathrm{g}_{\mathrm{cat}}{ }^{-1}$ representing respectively $39 \%$ and $17 \%$ of the values of the fresh nanocomposite. These results confirmed that the polymer loading and, what is more important, the loading of free acid sites remaining after hydrothermal treatment are very limited and so must be the residual activity left for successive runs. Low hydrothermal stability of the nanocomposites explains the unsatisfactory reusability properties. It is also very likely that the high activity observed for the initial runs is due in a large extension to the leached PSSA.

\subsubsection{Catalytic activity in the esterification of fatty acids}


This reaction is less demanding from the stability point of view than previous one as it is conducted in the presence of methanol and oil, less polar compounds, and at much lower temperature $(333 \mathrm{~K})$. It must be noticed that this reaction is biphasic (methanol is immiscible in oil), and the fatty acid (oleic acid) is majorly present in the oil phase.
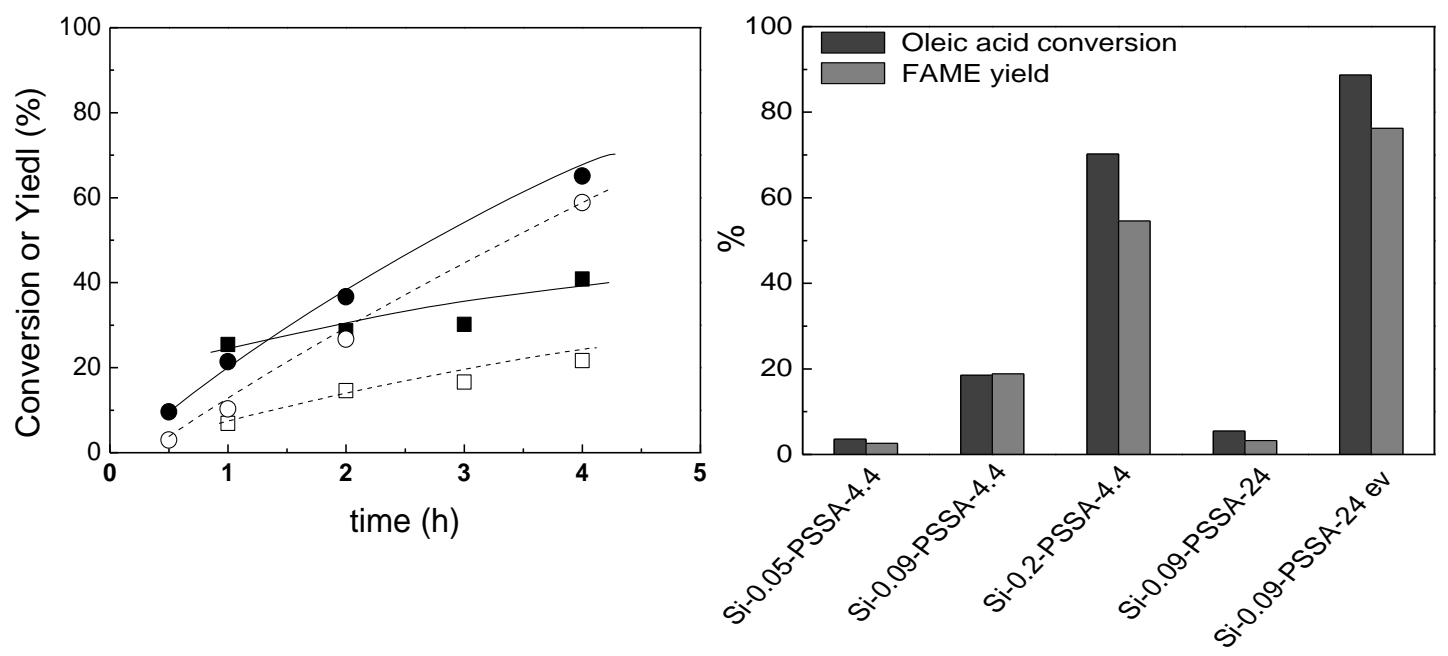

Figure 4. a) Oleic acid conversion (filled symbols) and FAME yield (empty symbols):

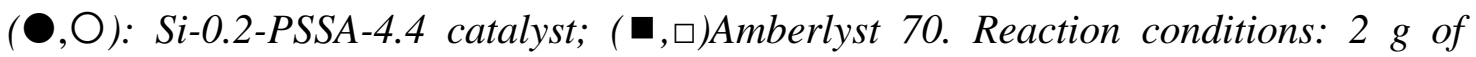
sunflower oil, methanol/oil mol ratio $=12$, FFA/oil wt. ratio $=0.05$, 3.3wt\% catalyst (catalyst/FFA wt. ratio $=0.4$ ), $333 \mathrm{~K} ;$ b) Oleic acid conversion and FAME yield for different catalysts. Reaction conditions: $2 \mathrm{~g}$ of sunflower oil, methanol/oil mol ratio $=12$, FFA/oil wt. ratio $=0.05,3.3 w t \%$ catalyst (catalyst $/ F F A w t$ ratio $=0.4$ ), $333 \mathrm{~K}$, $240 \mathrm{~min}$.

Figure 4a compares the activity of Si-0.2-PSSA-4.4 nanocomposite with that of commercial sulphonic resin (Amberlyst 70). It is clearly observed that nanocomposite compares favorably well with Amberlyst 70 especially when considering conversion of oleic acid and yield to FAME values at longer reaction time. Yield to FAME is in both cases smaller than conversion of oleic acid, very likely because part of the fatty acid is either chemisorbed or physisorbed within the pores network of the solid and accounted as converted. Figure $4 \mathrm{~b}$ compares the performance of the relevant nanocomposites studied in this article. Among the different catalyst tested, Si-0.2-PSSA-4.4 and Si-0.09PSSA-4.4-ev displayed the best performance. As in the case of furfural reaction, the presence of free acid sites in the nanocomposite is a key property but it is not the only feature having a role. Thus Si-0.2-PSSA-4.4, Si-0.09-PSSA-4.4 and Si-0.09-PSSA-24 
nanocomposites presented similar free acid sites loading and their performance is very alike. We hypothesized that textural porosity may have an important effect on the final activity because accessibility of voluminous free fatty acid to the active acid site is deeply related with the porosity of the solid.

Figure 5 compares the reusability behavior of the two most active catalysts in this reaction with those of Amberlyst 70. The improvement in the catalytic performance exhibited in the second run with respect to the first run is very likely related with the swelling by methanol experienced by the resin during the progress of the first run what may improve the accessibility of the reactant molecules to the acid sites present in the innermost region of the resin particles [26]. Amberlyst 70 is very stable along the six runs investigated. On the other hand, both nanocomposites are initially more active than Amberlyst 70. They are quite stable for five runs although an incipient deactivation was observed in the last sixth run investigated, more intense in the Si-0-09-PSSA.4.4-ev case. In any case they presented a higher stability in this reaction than for the furfural reaction. 
To gain information about the stability of the nacocomposites in the reaction mixture, leaching experiments were conducted by subjecting the nanocomposites to six consecutive leaching treatments consisting in contacting with methanol at $333 \mathrm{~K}$ for 4 hours. In practice after each run, the catalyst was centrifuged and the liquid phase removed. The solid was subsequently washed and centrifuged twice more with THF to remove the remaining methanol. The solid was dried at $323 \mathrm{~K}$ for $16 \mathrm{~h}$ and then a sample of solid was taken for elemental analysis before the next washing steps. The results of S concentration in the solid after the consecutive runs are shown in Figure 6.

Si-0.2-PSSA-4.4 nanocomposite presents a loss of S content close to $15 \%$ with respect to its initial content. However, in the next runs no relevant loss of $\mathrm{S}$ is observed and the nanocomposite presented an average concentration of ca. $2.2 \mathrm{mmolS} \cdot \mathrm{g}^{-1}$. This residual loss of polymer is in agreement with the stability of the activity observed for
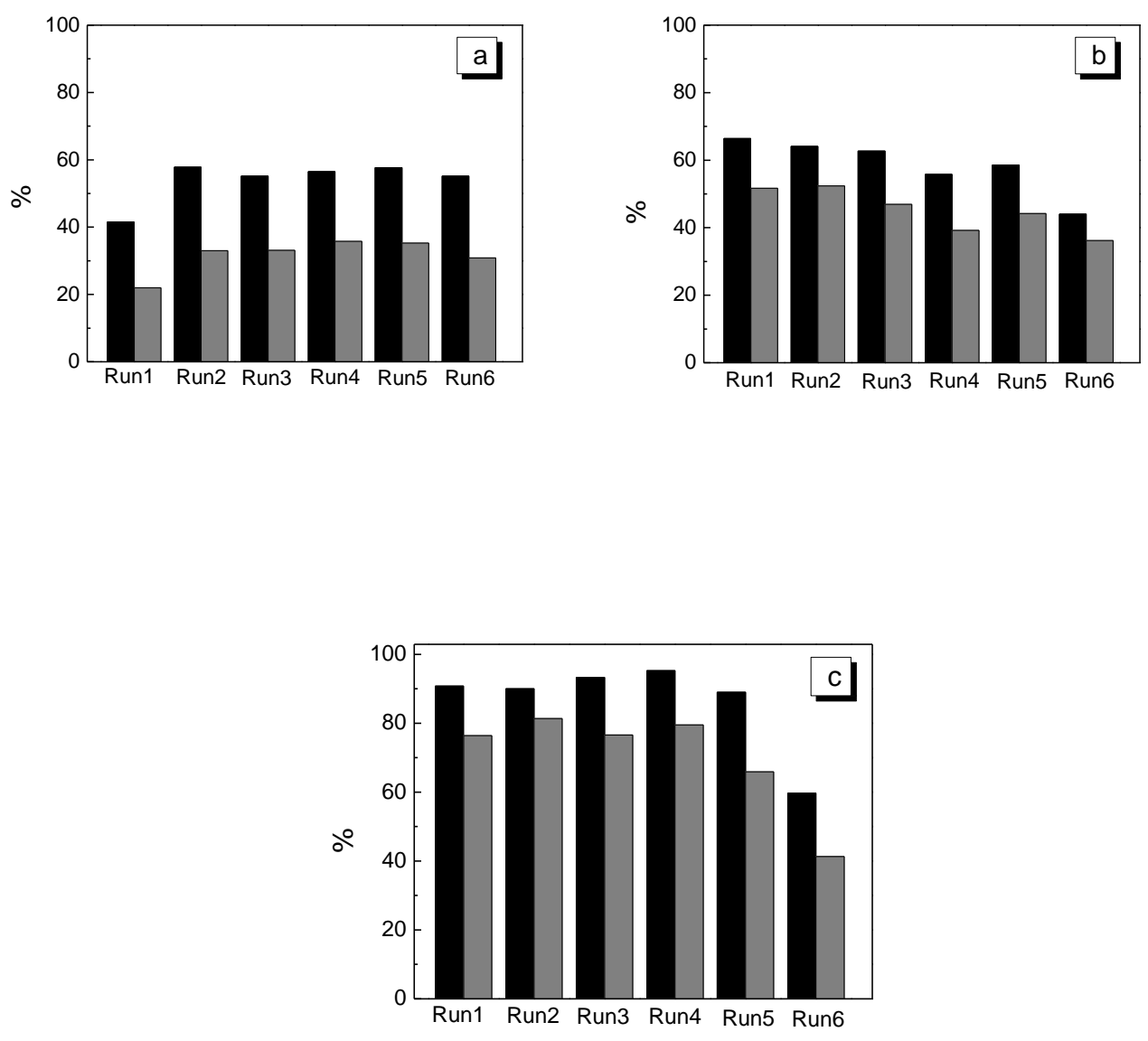

Figure 5. Reutilization of a) Amberlyst 70, b) Si-0.2-PSSA-4.4, c) Si-0.09PSSA-24-ev. Black bars correspond to oleic acid conversion and grey bars to FAME yield. Reaction conditions: $4 \mathrm{~g}$ of sunflower oil, methanol/oil mol ratio $=12$, FFA/oil wt. ratio $=0.05,3.3 w t \%$ catalyst $($ catalyst $/ F F A w t$ ratio $=0.4$ ), $333 \mathrm{~K}, 240 \mathrm{~min}$. 
this nanocomposite. On the other hand, the Si-0.09-PSSA-24-ev catalyst exhibited a remarkable loss of S loading (52\% of loss respect to its initial content). The loss for subsequent cycles is irrelevant, keeping a $\mathrm{S}$ concentration close to $2.0 \mathrm{mmolS} \cdot \mathrm{g}^{-1}$ in the next washing runs. It is worth mentioning that, in spite of the intense leaching of polymer observed during the first treatment for Si-0.09-PSSA-24-ev nanocomposite, no deactivation is observed in the catalytic activity. The lack of loss of polymer observed in successive runs is in agreement with the improved reusability properties displayed by this nanocomposite. The overall conclusion of these experiments is that in this reaction

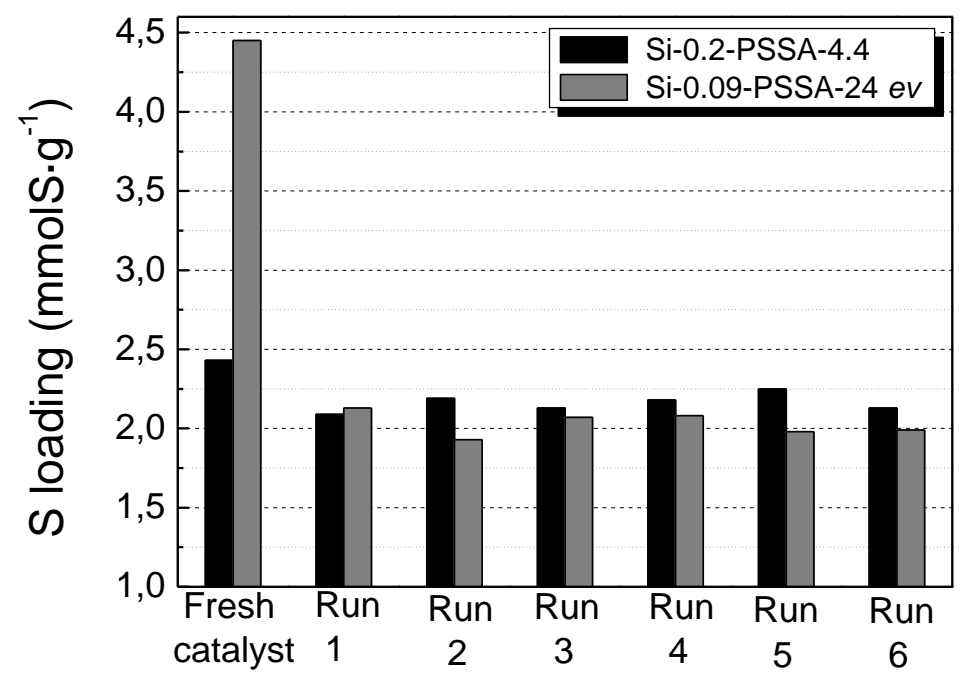

Figure 6. Sulphur concentration for different catalysts $\left(\mathrm{mmolS} \cdot \mathrm{g}^{-1}\right)$ after the consecutive runs of leaching. Reaction conditions: $40 \mathrm{mg}$ of catalyst, $5 \mathrm{~g}$ of methanol, 333K, 240 min.

leaching of polymer only takes place during the first run, corresponding to the most weakly retained polymer molecules (the latter are important in the Si-0.09-PSSA-24-ev sample). Other causes of deactivation must be taking place. Fouling of the surface by heavier molecules, formed by secondary reactions of fatty acids and triglycerides present in the oil, has been proposed to deactivate sulphonic silicas [27].

Another set of experiments, summarized in Figure 7, were conducted to estimate the contribution of the leached polymer to the overall activity. In practice the leached polymer was collected by contacting ca. $40 \mathrm{mg}$ of catalyst and ca. $5 \mathrm{~g}$ of methanol in the glass reactor and stirred for 4 hours at $333 \mathrm{~K}$. After this time, the reactor was cooled and the methanol solution was centrifuged to separate the liquid which contains the leached polymer from the catalyst. The remained solid was subsequently washed with methanol 
and centrifuged three more times. The methanol solutions from the treatment and the washings steps were accumulated in another Ace reactor and evaporated at $323 \mathrm{~K}$ for 16 $\mathrm{h}$ to dryness. The solid residue recovered (the dried leached polymer) was used as a soluble catalyst for the esterification reaction. This treatment was repeated three times with the same catalyst to estimate the contribution in three successive run. The results of the activity of these three cycles of leaching are shown in Figure 7 for both Si-0.2PSSA-4.4 and Si-0.09-PSSA-24-ev catalysts.
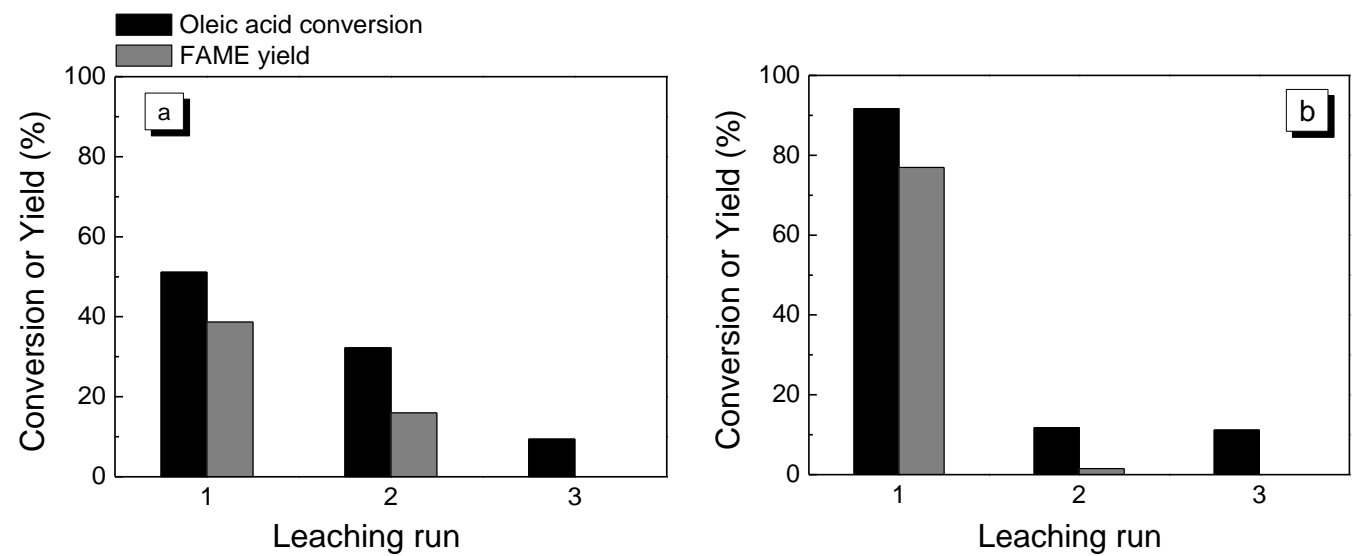

Figure 7. Oleic acid conversion and FAME yield of the leached species after in the consecutive leaching runs for the different catalysts: a) Si-0.2-PSSA-4.4; b) Si0.09-PSSA-24-ev. Reaction conditions: $4 \mathrm{~g}$ of sunflower oil, methanol/oil mol ratio $=12$, FFA/oil wt. ratio $=0.05,3.3 w t \%$ catalyst $($ catalyst $/ F F A w t$. ratio $=0.4), 333$ $K, 240 \mathrm{~min}$.

The results of the Figure 7 indicate that for both nanocomposites the contribution of leached species is very relevant for the first reaction cycle. In the case of Si-0.09-PSSA-4.4 the homogenous contribution is still important for the second run but negligible for the third run. In the case of Si-0.09-PSSA-24-ev nanohybrid the homogeneous contribution is already negligible in the second run. These results are in agreement with those in Figure 6 that showed that leaching was more important in the first cycles.

It is noticeable that the homogeneous contribution for the first run, for both catalysts, is quite similar to the overall activity displayed by the solid catalyst. This may erroneously suggest that for the first run all the activity is due to the leached species: the leaching of the polymer is minor in the second and successive runs and the activity of the solid in these reaction cycles is still as high as that displayed in the first cycle. 
Therefore this type of experiments seems to overestimate the leaching contribution during the first run. It may be possible that leaching rate is not fast but of the same order of the reaction rate. The experiments conducted in Figure 7 in practice measured the contribution of the species leached after $4 \mathrm{~h}$ of leaching treatment and does not exactly represent the real situation during the course of the reaction. The homogeneous contribution can be low at the beginning as leaching has not been intense yet, and rises upon reaction time along with the heterogeneous contribution.

Besides we have also to take into account that removal of weakly retained PSSA polymer allows the access of the reactants to fresh anchored sulphonic polymer that explains why in successive cycles, when there is no significant leaching, the solid is still very active. In summary, a more correct conclusion is that contribution of leaching is only important for the first run whereas the activity measured in second and successive runs are due to the firmly retained PSSA.

\section{Conclusions}

Polymer-SiO $\mathrm{S}_{2}$ nanocomposites involving PSSA acid polymer can be prepared by sol-gel methodologies; an amino functionalized organosilane (APTES) is required to provide the anchoring sites for the retention of the acid polymer by the silica particles network. The acid sites loading present in the nanocomposites increases upon the N/Si ratio used for the synthesis (larger proportion of APTES) up to an at. N/Si ratio $=0.09$. Beyond this value, acid sites loading did not increase further. The utilization of methanol as solvent to conduct the synthesis instead of water did not result in an improvement of the acid sites loading, neither did a long period of aging. The slow evaporation, before the drying process, of the mother liquor resulting from the sol-gel synthesis results in nanocomposites with a very high concentration of acid polymer loading.

When this type of nanocomposites is used as catalyst for the reaction of transformation of xylose to furfural, a reaction representative of those conducted at relatively high temperatures in water medium, they significantly deactivated, mainly due to the extensive leaching of the acid polymer. The leaching is driven by the harsh conditions at which the reaction is conducted (aqueous environment at $453 \mathrm{~K}$ ). 
On the contrary when used for the esterification of oleic acid with methanol to yield biodiesel, a reaction conducted in organic medium and at milder temperatures $(333 \mathrm{~K})$, the deactivation by leaching is significantly prevented and is only important during the first contact of the catalyst with the reaction medium. Once that the more weakly retained PSSA molecules are removed in the first cycle, those more stable remained and are not leached in successive runs. Consequently this type of nanocomposites can be potentially used as catalysts for reactions conducted at moderate temperatures, although for the specific case here presented, catalysts deactivated by fouling.

\section{Acknowledgments}

Financial support from the Spanish Ministry of Economy and Competitiveness is gratefully acknowledged (project CTQ2012-38204-C03-01 and predoctoral grant for M.J.C.M). JJCP also thanks Energy and Environment Postgraduate Studies program in Spain for a IBERDROLA Foundation Scholarships

\section{References}

[1] T.J. Dickerson, N.N. Reed, K.D. Janda, Soluble polymers as scaffolds for recoverable catalysts and reagents, Chem. Rev., 102 (2002) 3325-3344

[2] A. Corma Canos, S. Iborra, A. Velty, Chemical routes for the transformation of biomass into chemicals, Chem. Rev., 107 (2007) 2411-2502.

[3] D.M. Alonso, S.G. Wettstein, J.A. Dumesic, Gamma-valerolactone, a sustainable platform molecule derived from lignocellulosic biomass, Green Chem., 15 (2013) 584-595.

[4] S.G. Wettstein, D. Martin Alonso, E.I. Gürbüz, J.A. Dumesic, A roadmap for conversion of lignocellulosic biomass to chemicals and fuels, Curr. Opin. Chem. Eng., 1 (2012) 218-224.

[5] S. Van De Vyver, J. Thomas, J. Geboers, S. Keyzer, M. Smet, W. Dehaen, P.A. Jacobs, B.F. Sels, Catalytic production of levulinic acid from cellulose and other biomass-derived carbohydrates with sulphonated hyperbranched poly(arylene oxindole)s, Energy Environ. Sci., 4 (2011) 3601-3610.

[6] M. López Granados, A.C. Alba-Rubio, I. Sádaba, R. Mariscal, I. MateosAparicio, A. Heras, Poly(styrenesulphonic) acid: An active and reusable acid catalyst soluble in polar solvents, Green Chem., 13 (2011) 3203-3212.

[7] H. Zou, S. Wu, J. Shen, Polymer/Silica Nanocomposites: Preparation, characterization, propertles, and applications, Chem. Rev., 108 (2008) 3893-3957.

[8] A. Martín, G. Morales, F. Martínez, R. Van Grieken, L. Cao, M. Kruk, Acid hybrid catalysts from poly(styrenesulfonic acid) grafted onto ultra-large-pore SBA-15 
silica using atom transfer radical polymerization, J. Mater. Chem., 20 (2010) 80268035.

[9] R. Tamaki, Y. Chujo, Synthesis of polystyrene and silica gel polymer hybrids utilizing ionic interactions, Chem. Mater., 11 (1999) 1719-1726.

[10] I. Sádaba, M. Ojeda, R. Mariscal, M. López Granados, Silicapoly(styrenesulphonic acid) nanocomposites for the catalytic dehydration of xylose to furfural, Appl. Catal. B: Environ., 150-151 (2014) 421-431.

[11] N. Alonso-Fagúndez, V. Laserna, A.C. Alba-Rubio, M. Mengibar, A. Heras, R. Mariscal, M. López Granados, Poly-(styrene sulphonic acid): An acid catalyst from polystyrene waste for reactions of interest in biomass valorization, Catal. Today, 234 (2014), 285-294.

[12] M.J. Campos, R. Mariscal, M. López. Granados, Synthesis of silica xerogelpoly(styrene sulphonic acid) nanocomposites as acid catalysts: effects of temperature and polymer concentration on their textural and chemical properties, J. Sol-Gel Sci.Technol., 75 (2015) 164-179.

[13] M. J. Campos Molina, R. Mariscal, M. Ojeda, M. López Granados, Cyclopentyl methyl ether: A green co-solvent for the selective dehydration of lignocellulosic pentoses to furfural, Bioresour. Technol., 126 (2012) 321-327.

[14] J.J. Bozell, G.R. Petersen, Technology development for the production of biobased products from biorefinery carbohydrates, Green Chem., 12 (2010) 539-554.

[15] J.A. Melero, J. Iglesias, G. Morales, Heterogeneous acid catalysts for biodiesel production: current status and future challenges, Green Chem., 11 (2009) 1285-1308.

[16] M. Di Serio, R. Tesser, L. Pengmei, E. Santacesaria, Heterogeneous catalysts for biodiesel production, Energy and Fuels, 22 (2008) 207.

[17] C. García-Sancho, R. Moreno-Tost, J.M. Mérida-Robles, J. SantamaríaGonzález, A. Jiménez-López and P. Maireles-Torres, Niobium-containing MCM-41 silica catalysts for biodiesel production, Applied Catalysis B: Environmental, 108-109 (2011) 161.

[18] X. Shi, Y. Wu, H. Yi, G. Rui, P. Li, M. Yang, G. Wang, Selective preparation of furfural from xylose over sulfonic acid functionalized mesoporous Sba-15 materials, Energies, 4 (2011) 669-684.

[19] X. Wang, S. Cheng, J.C.C. Chan, Propylsulfonic acid-functionalized mesoporous silica synthesized by in situ oxidation of thiol groups under template-free condition, J. Phys. Chem. C, 111 (2007) 2156-2164.

[20] J.D. Wright, N.A.J.M. Sommerdijk, Sol-Gel Materials Chemistry and applications, CRC Press.

[21] C.J. Brinker, D.M. Smith, R. Deshpande, P.M. Davis, S. Hietala, G.C. Frye, C.S. Ashley, R.A. Assink, Sol-gel processing of controlled pore oxides, Catal. Today, 14 (1992) 155-163.

[22] S. Chen, S. Hayakawa, Y. Shirosaki, E. Fujii, K. Kawabata, K. Tsuru, A. Osaka, Sol-gel synthesis and microstructure analysis of amino-modified hybrid silica nanoparticles from aminopropyltriethoxysilane and tetraethoxysilane, J. Amer. Ceram. Soc., 92 (2009) 2074-2082.

[23] G. Stephen Caravajal, D.E. Leyden, G.R. Quinting, G.E. Maciel, Structural characterization of (3-aminopropyl)triethoxysilane-modified silicas by silicon-29 and carbon-13 nuclear magnetic resonance, Anal. Chem., 60 (1988) 1776-1786.

[24] C.H. Chiang, N.I. Liu, J.L. Koenig, Magic-angle cross-polarization carbon 13 NMR study of aminosilane coupling agents on silica surfaces, J. Colloid Interface Sci., 86 (1982) 26-34. 
[25] J.E. Sarneski, H.L. Surprenant, F.K. Molen, C.N. Reilley, Chemical shifts and protonation shifts in carbon-13 nuclear magnetic resonance studies of aqueous amines, Anal. Chem., 47 (1975) 2116-2124.

[26] D.M. Alonso, M. López Granados, R. Mariscal, A. Douhal, Polarity of the acid chain of esters and transesterification activity of acid catalysts, J. Catal., 262 (2009) 1826.

[27] A.C. Alba-Rubio, F. Vila, D.M. Alonso, M. Ojeda, R. Mariscal, M. López Granados, Deactivation of organosulfonic acid functionalized silica catalysts during biodiesel synthesis, Appl. Catal. B: Environ., 95 (2010) 279-287. 